Copyright (C1996, American Institute of Aeronautics and Astronautics, Inc.

AIAA Meeting Papers on Disc, January 1996

A9618553, AIAA Paper 96-0593

\title{
The propagation of a vortex ring through multiple liquid interfaces in microgravity
}

\author{
L. P. Bernal \\ Michigan Univ., Ann Arbor \\ P. Maksimovic \\ Michigan Univ., Ann Arbor
}

\section{AIAA 34th Aerospace Sciences Meeting and Exhibit, Reno, NV Jan 15-18, 1996}

\begin{abstract}
We report experimental observations of the propagation of a vortex ring through two liquid interfaces separating immiscible liquids. The experiments examine the effect of vortex ring strength and the thickness of the middle layer on the evolution of the flow. A wide range of phenomena is observed. In particular, we show that at some conditions the vortex ring enters the top layer without bringing with it fluid from the middle layer. At higher Weber numbers, the vortex ring engulfs fluid from the middle layer and forms a drop of liquid from the lower layer coated with fluid from the middle layer. (Author)
\end{abstract}




\title{
The Propagation of a Vortex Ring through Multiple Liquid Interfaces in Microgravity
}

\author{
L.P. Bernal *, P. Maksimovic ${ }^{\dagger}$ \\ University of Michigan \\ Ann Arbor, MI. 48109-2118
}

\begin{abstract}
We report experimental observations of the propagation of a vortex ring through two liquid interfaces separating immiscible liquids. The experiments examine the effect of vortex ring strength and the thickness of the middle layer on the evolution of the flow. A wide range of phenomena is observed. In particular, we show that at some conditions the vortex ring enters the top layer without bringing with it fluid from the middle layer. At higher Weber number the vortex ring engulfs fluid from the middle layer and forms a drop of liquid from the lower layer coated with fluid from the middle layer.
\end{abstract}

\section{Introduction}

This paper examines experimentally the propagation of a vortex ring through several layers of immiscible liquids. The flow configuration is shown schematically in Figure 1. It consists of a vortex ring propagating upward through two interfaces. If the vortex ring has sufficient impulse the collision of the vortex ring with the interfaces will result in the formation of a drop. The liquid inside the drop is fluid being carried along by the vortex ring and therefore is fluid from the lower layer. The three layer structure of the initial flow configuration suggests that the resulting drop might have a similar structure with fluid from each layer forming coating layers on the original drop. This relatively simple flow system captures many important processes relevant to the evolution of fluid interfaces in microgravity and in other flow systems.

The study of the interaction of a vortex ring with multiple fluid interfaces is motivated in part by problems in encapsulation technology. The goal of this technology is to produce small drops of a drug solution coated with a membrane of suitable chemical and biological properties. The fluid dynamic processes encountered in this technology have been reviewed by Kendall et al. ${ }^{1}$ Lin and $W$ ang $^{2}$ proposed an encapsulation system using a droplet generator based on the Rayleigh instability of a liquid column. The present experiments of a vortex ring propagating through multiple fluid interfaces provide new insights on these

\footnotetext{
* Associate Professor, Department of Aerospace Engineering. Senior Member AIAA.

* Research Assistant, Department of Aerospace Engineering.

Copyright (C) 1996 American Institute of Aeronautics and Astronautics, Inc. All rights reserved
}

problems and should contribute to the development of improved encapsulation technology.

Bernal et al ${ }^{3}$ studied the interaction of vortex rings with a single liquid interface. They considered the case of small density change across the interface. These experiments showed that at sufficiently high Froude and Weber number the vortex ring penetrates the interface and forms a drop. Before breakup the interface is highly stretched and vorticity is left behind along the liquid column. The location where the interface break depends on the strength of the vortex ring. It occurs near the vortex ring at high Weber number and away from the vortex ring, near the undisturbed interface at low Weber number. They observed the formation of satellite drops during the interaction that are associated with concentrations of vorticity along the stretched interface. They showed good agreement between numerical and experimental results. Numerical results at several Froude and Weber numbers showed that in the capillary limit drops form at Weber number greater than $\approx 2$. An interesting observation is the formation of a reflected vortex ring propagating in the direction opposite to the drop motion.

In this paper we extend the results of Bernal et $a l^{3}$ by considering the case of three liquid layers. The flow is characterized by the vortex ring diameter, $a$, and propagation speed, U. The size of the resulting drop scales with the vortex ring diameter $a$. The interfaces are characterized by the densities $\rho_{1}, \rho_{2}$ and $\rho_{3}$; the viscosities $\mu_{1}, \mu_{2}$ and $\mu_{3}$; and the interfacial tensions $\sigma_{12}$ and $\sigma_{23}$. The flow evolution depends on fluid inertia, viscosity and surface tension. Therefore the nondimensional numbers defining the problem are the Weber number, the Froude number and the Reynolds number which in this case can be defined based on the liquid properties of each interface. These parameters defined based on the fluid properties of the lower layer and interface are:

$$
W e_{1}=U \sqrt{\frac{\rho_{1} a}{\sigma_{12}}}, \quad F_{1}=\frac{U}{\sqrt{\frac{\rho_{1}-\rho_{2}}{\rho_{!}} g_{a}}}, \quad \operatorname{Re}_{1}=\frac{\rho_{1} U a}{\mu_{1}},
$$

respectively. In addition, the density ratio $r_{12}=\rho_{2} / \rho_{1}$ and $r_{23}=\rho_{3} / \rho_{2}$, as well as the viscosity ratio $\lambda_{12}=\mu_{2} / \mu_{1}$ and $\lambda_{23}=\mu_{3} / \mu_{2}$ must be specified.

The relative magnitude of the gravitational acceleration compared to the surface tension force is characterized by the Bond number which we define here as the ratio of the Weber and 
Froude number. In the present experiments there is a different Bond number for each interface. Thus,

$$
\mathrm{Bo}_{1}=\frac{\mathrm{We}_{1}}{\mathrm{Fr}_{1}}=\sqrt{\frac{\rho_{1}-\rho_{2}}{\sigma_{12}} \mathrm{ga}},
$$

and

$$
\mathrm{Bo}_{2}=\frac{\mathrm{We}_{2}}{\mathrm{Fr}_{2}}=\sqrt{\frac{\rho_{3}-\rho_{2}}{\sigma_{23}} \mathrm{ga}} .
$$

In microencapsulation systems a typical Bond number is small because the drop diameter is small and, therefore, even in a $1 \mathrm{~g}$ environment with large density change across the interface the flow evolution is surface tension dominated. This flow regime can be examined at a much larger scale in microgravity because $g$ is very small $\left(\approx 10^{-5}\right)$. In the present experiment we explore the surface tension dominated small Bond number regime using liquids of matched densities (i.e. small density difference) and reasonably large drop size.

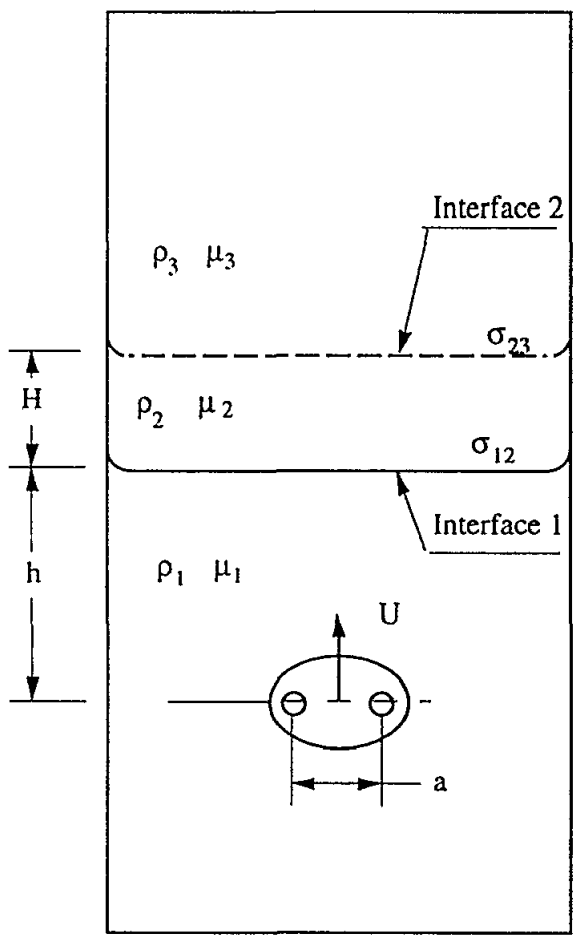

Figure 1. Schematic diagram of the flow configuration.

\section{Flow Facility and Experimental Techniques}

The experiments were conducted in a small container $115 \times 115 \times 300 \mathrm{~mm}$. For the present experiments the liquids used were: a mixture of water and methanol with density 0.950 $\mathrm{gr} / \mathrm{cm}^{3}$ in the lower layer, silicon oil (Dow Corning 200,10cs) with density $0.946 \mathrm{gr} / \mathrm{cm}^{3}$ in the middle layer, and a mixture of water and methanol with density $0.940 \mathrm{gr} / \mathrm{cm}^{3}$ in the top layer. The density and viscosity ratios for this fluid system are:

$$
r_{12}=0.996, r_{23}=0.994,
$$

and

$$
\lambda_{12}=5.28, \lambda_{23}=0.195 \text {. }
$$

The interfacial tension was measured using the capillary rise technique. ${ }^{4}$ The measured values of the interfacial tension for this system were

$$
\sigma_{12}=20.0 \mathrm{mN} / \mathrm{m}, \quad \sigma_{23}=15.5 \mathrm{mN} / \mathrm{m} .
$$

The same liquids were used in all the experiments reported here.

A piston-type vortex ring generator was used in the experiments. The vortex ring is produced by the motion of a piston inside a cylinder installed at the bottom of the container. The piston diameter is $1.27 \mathrm{~cm}$ and the length of the cylinder $1.27 \mathrm{~mm}$. At the end of the stroke the piston surface is flush with the bottom of the container resulting in a smooth flat surface during the test. The piston is driven by a pneumatic actuator. The input air pressure to the pneumatic actuator determines the speed of the piston and consequently the initial speed, $U$, of the vortex ring. A pressure regulator and a solenoid valve were used to control the input air pressure and the start of the fluid motion, respectively.

In the experiments the liquid layer structure was varied by changing the thickness of the middle layer, $\mathrm{H}$. The thickness of the lower layer was kept constant at $4 \mathrm{~cm}$. The thickness of the upper layer was large enough to avoid interaction of the fluid motion with the free surface on top. As described above, the relative magnitude of surface tension effects compared to surface tension at each interface is given by the Bond number which for the present experiments were:

$$
\mathrm{Bo}_{12}=0.56, \quad \mathrm{Bo}_{23}=0.78 \text {. }
$$

Although these values are less than one, they are not small indicating that the effect of gravitational acceleration is not negligible.

To visualize the flow, a fluorescent dye (fluorescein $\approx 3 \mathrm{ppm}$ ) was added to the fluid in the vortex ring generator. The flow field was illuminated with a thin sheet of light derived from an Argon-Ion laser. The light sheet was positioned along the axis of symmetry of the flow. The fluid motion was recorded using a $35 \mathrm{~mm}$ SLR camera and a video system. Measurements of the position of the vortex ring and of the deformation of the interface were obtained using the video images. 
Copyright (c)1996, American Institute of Aeronautics and Astronautics, Inc.

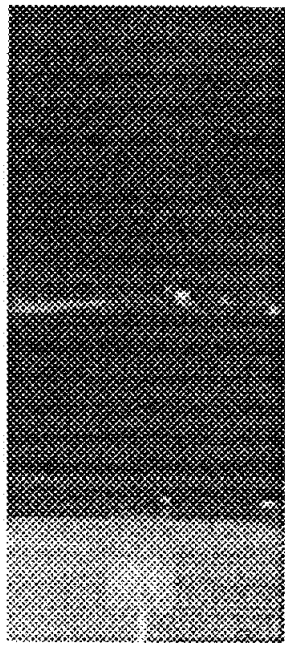

(a) $t=0.078 \mathrm{~s}$

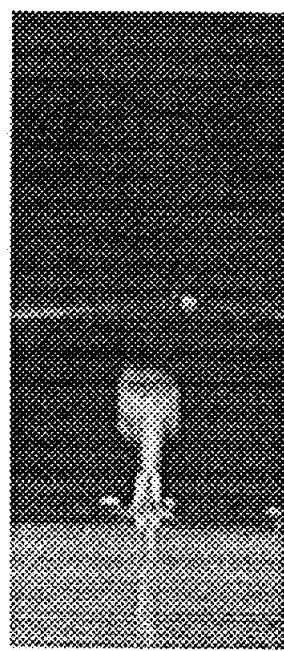

(b) $\mathrm{t}=0.218 \mathrm{~s}$

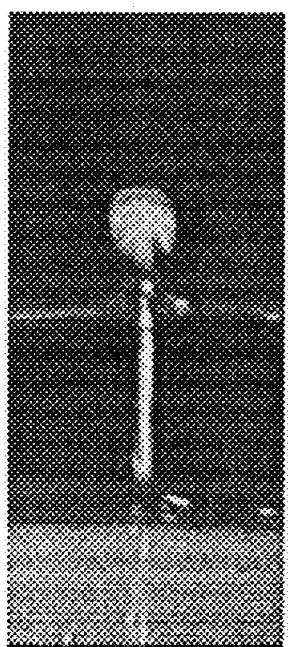

(c) $t=0.378 \mathrm{~s}$

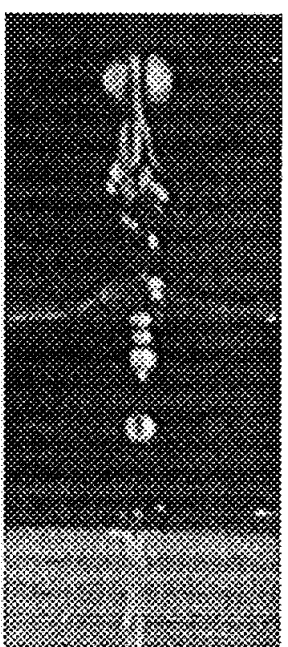

(d) $\mathrm{t}=0.587 \mathrm{~s}$

Figure 2. Propagation of a vortex ring through two fluid interfaces. Froude number $\mathrm{Fr}_{1}=10$, Weber number, $\mathrm{We}_{1}=$ 7, Reynolds number 1920.

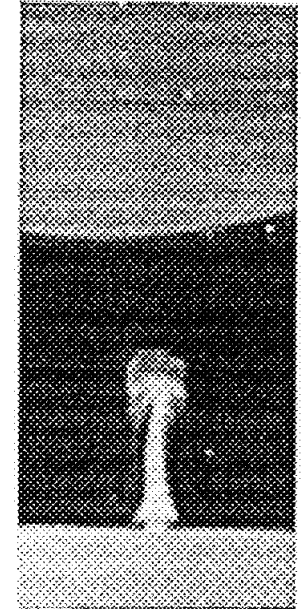

(a) $\mathrm{t}=0.158 \mathrm{~s}$

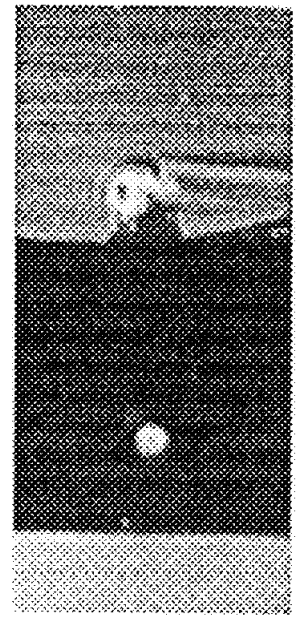

(d) $\mathrm{t}=0.458 \mathrm{~s}$

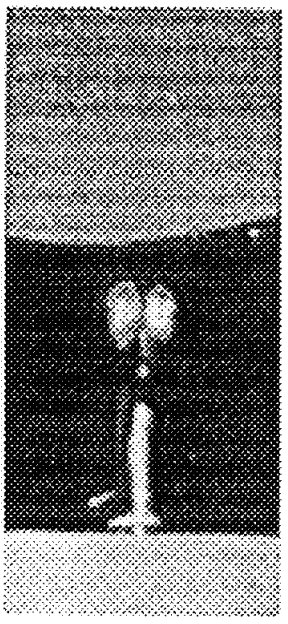

(b) $\mathrm{t}=0.218 \mathrm{~s}$

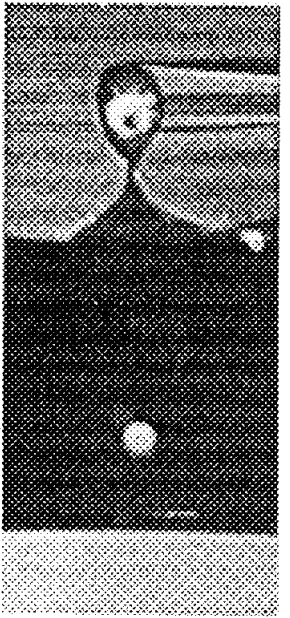

(e) $\mathrm{t}=0.988 \mathrm{~s}$

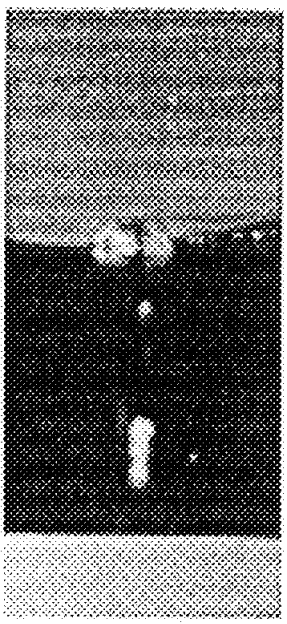

(c) $\mathrm{t}=0.318 \mathrm{~s}$

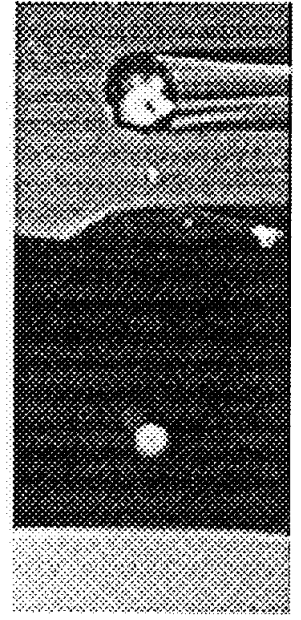

(f) $\mathrm{t}=1.118 \mathrm{~s}$

Figure 3. Propagation of a vortex ring through two fluid interfaces. Froude number $F_{r_{1}}=18$, Weber number, We $e_{1}=$ 12, Reynolds number 3373. 


\section{Results and Discussion}

Figure 2 shows the evolution of a relatively weak vortex ring. The Froude and Weber number based on the lower interface properties are 10 and 7 respectively. The thickness of the middle layer is $\mathrm{H} / \mathrm{a}=3.7$. The initial evolution is similar to that reported by Bernal et al $^{3}$ for a single interface. In this case however, the vortex ring reaches the second interface before the liquid column breaks and a drop forms. The third image in the sequence captures the flow after the vortex ring has penetrated the second interface and the liquid column breakup. The final image in the sequence shows the vortex ring in the liquid layer on top. At these conditions the vortex ring does not carry any silicone oil from the middle layer. Because the top and bottom layer are made of the same components, namely water and methanol, the vortex ring structure that forms in the top liquid layer is not a drop. In this case fluid from the lower layer is transferred to the top layer without a silicone oil coating. The pictures also show the formation of smaller satellite drops below the main vortical region.

Figure 3 shows the evolution of a stronger vortex ring and a larger depth of the middle layer. The Froude and Weber number based on the lower interface properties are 18 and 12 respectively. The thickness of the middle layer is $\mathrm{H} / \mathrm{a}=5.9$. In this case the vortex ring penetrates the interface and forms a drop of liquid from the lower layer in the middle layer. The drop forms before reaching the second interface in the third image. The last three images show that the interaction with the second interface results in the formation of a drop of dyed fluid in the top layer covered by a layer of silicone oil. The dyed liquid originated in the lower layer while the silicone oil originated in the middle layer This "drop-within-a-drop" structure indicates that at these flow conditions the drop engulfs a significant amount of silicone oil as it moves through the middle layer.

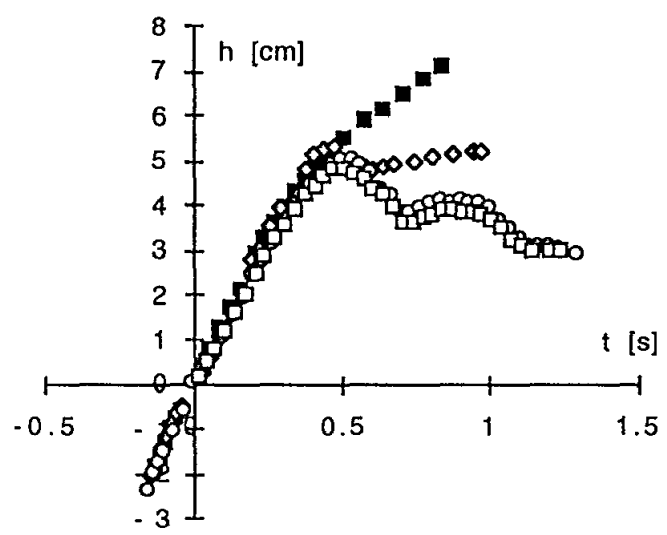

Figure 4. Vortex ring position as a function of time for Weber number 3.9 and Reynolds number 1053. $\mathrm{I}, \mathrm{H}=2.6$ $\mathrm{cm} ; \odot, \mathrm{H}=4.7 \mathrm{~cm} ; \mathrm{O}, \mathrm{H}=6.6 \mathrm{~cm} ; \square, \mathrm{H}=7.5$ $\mathrm{cm}$;

The effect of the depth of the middle layer for a fixed vortex ring speed is illustrated in Figure 4. In this case the Weber number is $\mathrm{We}_{\mathrm{l}}=3.9$. For the smaller middle layer depth $\mathrm{H}=$
$2.6 \mathrm{~cm}$, the vortex ring propagates through both interfaces. Although the speed decreases as the vortex ring moves in the top layer, the speed is substantial even at the last point measured. In contrast, as the depth of the middle layer is increased the vortex ring motion changes significantly. At a depth of $4.7 \mathrm{~cm}$ the vortex ring still penetrates the second interface but its speed is substantially reduced in the process. At even larger depths, $\mathrm{H}=$ $6.6 \mathrm{~cm}$ and $7.5 \mathrm{~cm}$, the vortex ring no longer penetrates the second interface. This result is striking since as the depth of the middle layer is increased a lighter liquid is replaced by a heavier liquid and, consequently, the effect of gravitational acceleration is to increase the vortex ring speed. The observed slow down of the vortex ring for increased thickness of the middle layer could be a viscous effect at the drop surface. The Reynolds number based on the lower layer properties is 1053 and, based on the silicone oil properties is 200 . These values support the observation that viscous effects at the drop surface play an important role in the evolution of the flow.

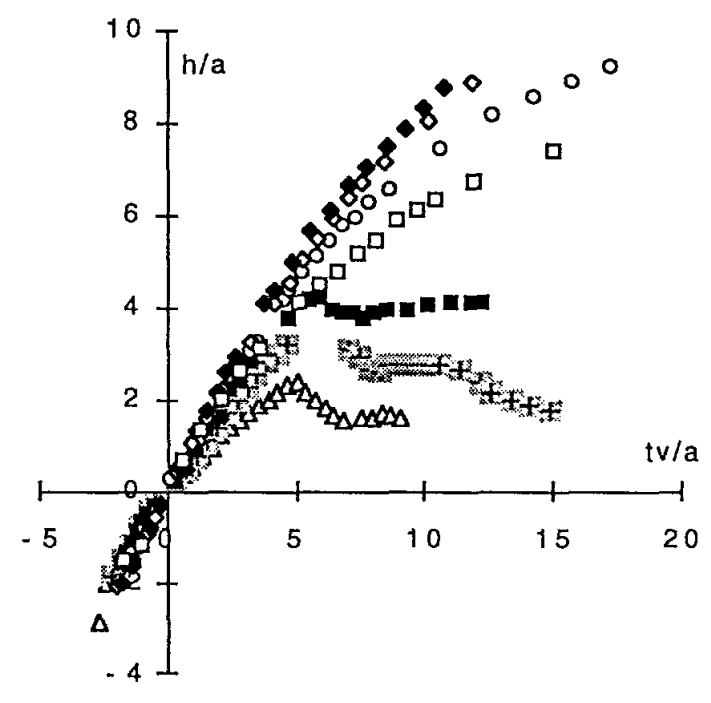

Figure 5. Vortex ring position, $\mathrm{h} / \mathrm{a}$, as a function of time, $\mathrm{t} / \mathrm{a}$, for $\mathrm{H} / \mathrm{a}=3.7 . \Delta, \mathrm{We}_{1}=3, \mathrm{Re}_{1}=833 ; \boxplus, \mathrm{We}_{1}=$ $3.3 \operatorname{Re}_{1}=897 ; \mathrm{We}_{1}=3.8 \mathrm{Re}_{1}=1053 ; \mathrm{O}, \mathrm{We}_{1}=$ $5 \mathrm{Re}_{1}=1362 ; \diamond, \mathrm{We}_{1}=5.5 \mathrm{Re}_{1}=1498 ; \diamond, \mathrm{We}_{1}=$ $8 \mathrm{Re}_{1}=2206 ; \square, \mathrm{We}_{1}=12.6 \operatorname{Re}_{1}=3453$.

The effect of Weber number on the flow evolution for fixed middle layer depth is shown in Figures 5 and 6 . Figure 5 is a plot of $\mathrm{h} / \mathrm{a}$ as a function of non dimensional time for a middle layer depth of $4.7 \mathrm{~cm}$ which corresponds to a non dimensional value of $\mathrm{H} / \mathrm{a}=3.7$. For a Weber number less than 3.8 , the vortex ring enters the middle layer but does not penetrate the second interface, At a-Weber number of 3.8 the vortex ring enters the top liquid layer but the speed is very low. For Weber number larger than 3.8 the vortex ring penetrates the second interface. In these cases the yortex ring in the top liquid layer does not carry a layer of silicone oil from the middle layer. 


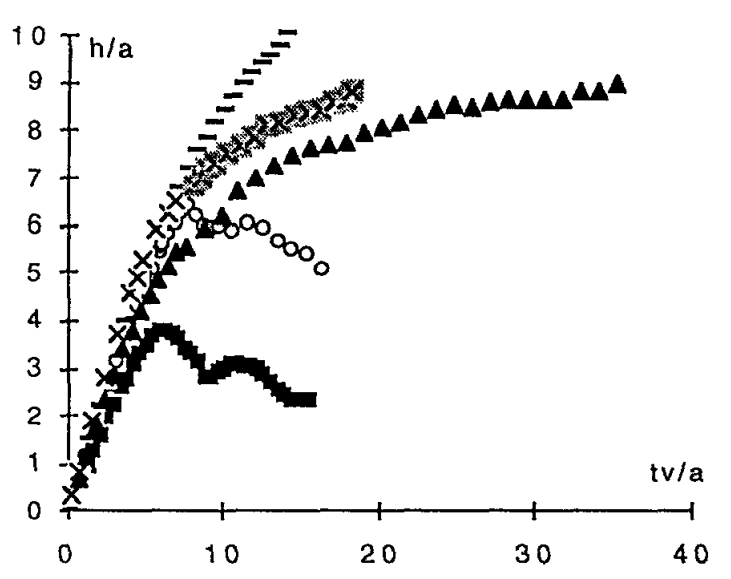

Figure 6. Vortex ring position, $\mathrm{h} / \mathrm{a}$, as a function of time, $\mathrm{tv} / \mathrm{a}$, for $\mathrm{H} / \mathrm{a}=5.9 . \mathrm{W}, \mathrm{We}_{\mathrm{I}}=3.8, \mathrm{Re}_{1}=1053 ; 0, \mathrm{We}_{1}$ $=5 \operatorname{Re}_{1}=1362 ;-, \mathrm{We}_{1}=6.4 \mathrm{Re}_{1}=1746 ; \mathrm{X}, \mathrm{We}_{1}$ $=9.2 \operatorname{Re}_{1}=2518$ (silicone oil and dye); $\otimes, \mathrm{We}_{1}=$ $9.2 \operatorname{Re}_{1}=2518$ (Silicone oil); $\Delta, \mathrm{We}_{1}=11.6 \mathrm{Re}_{1}=$ 3453 .

Figure 6 is a plot of the nondimensional position of the vortex ring, $h / a$, as a function of nondimensional time for a depth of the middle layer $\mathrm{H} / \mathrm{a}=5.9$. This plot illustrates the wide range of interactions found in this study. At low Weber number $\left(W_{1}=3.8\right)$, the vortex ring does not penetrate the second interface and eventually moves down by the effect of buoyancy. At a slightly higher Weber number $\left(\mathrm{We}_{1}=5\right)$, the vortex ring penetrates the interface and, due to buoyancy, slows and moves down. At a $W e_{1}=6.4$ the vortex ring has enough kinetic energy to continue the upward motion for the entire duration of the test. The evolution changes at $\mathrm{We}_{1}=9.2$. In this case the vortex ring engulfs a large amount of fluid from the middle layer into the top layer to form a drop. However, this configuration is not stable and the dyed fluid is ejected and a drop of only silicone oil forms in the top layer. The final case at a Weber number of $\mathrm{We}_{1}=11.6$ shows the evolution when the final outcome of the interaction is a drop of fluid from the lower layer coated with silicone oil from the middle layer. In this case the speed of the vortex ring is significantly reduced as it propagates through the upper two layers.

\section{Conclusions}

The main conclusions of this study can be summarized as follows:

- For small thickness of the silicone oil layer and a low viscosity silicone oil the interaction results in the collapse of the entire liquid layer structure.

- Three flow regimes have been identified when the vortex ring penetrates the second interface. 1) At low Weber number, the vortex ring propagates into the upper layer but does not carry a outer layer of silicone oil. 2) At high values of the Weber number, the vortex ring propagates into the upper layer with an outer layer (coating) of silicone oil. 3) At some intermediate values of the Weber number, the coated drop structure is unstable. In some cases a single drop of silicone oil forms.

- The Weber number value for the boundaries between these regimes depend on the depth of the middle layer.

\section{References}

1. J.M. Kendall, M. Chang, \& T.G. Wang, "Fluid and chemical dynamics relating to encapsulation technology," AIP Proceeding 197, Int'l Colloquium on drops and bubbles, Monterey, CA. 1988.

2. K.C. Lin \& T.G. Wang, "A novel method for producing microspheres with semipermeable polymer membranes," AIAA paper 92-0118, AIAA 30nd Aerospace Sciences Meeting \& Exhibit. Reno, NV. January 6-9, 1992.

3. L.P. Bernal, P. Maksimovic, F. Tounsi \& G. Tryggvason."An experimental and numerical investigation of drop formation by vortical flows in microgravity." AIAA paper 94-0244, AIAA 32nd Aerospace Sciences Meeting \& Exhibit. Reno, NV. January 10-13, 1994.

4. S.B. Reddy Karri and V.K. Mathur. Measurement of interfacial tension of immiscible liquids of equal density. AIChE Journal, vol 34 (1988) no 1, p155. 


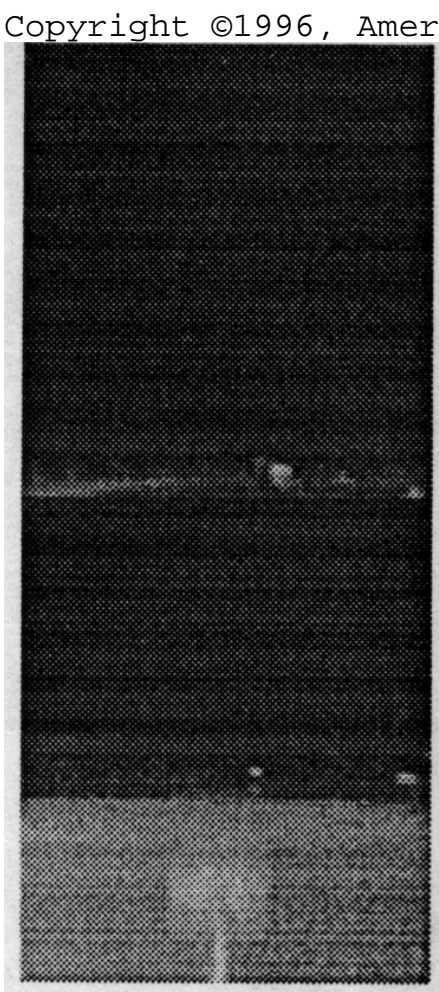

(a) $t=0.078 \mathrm{~s}$

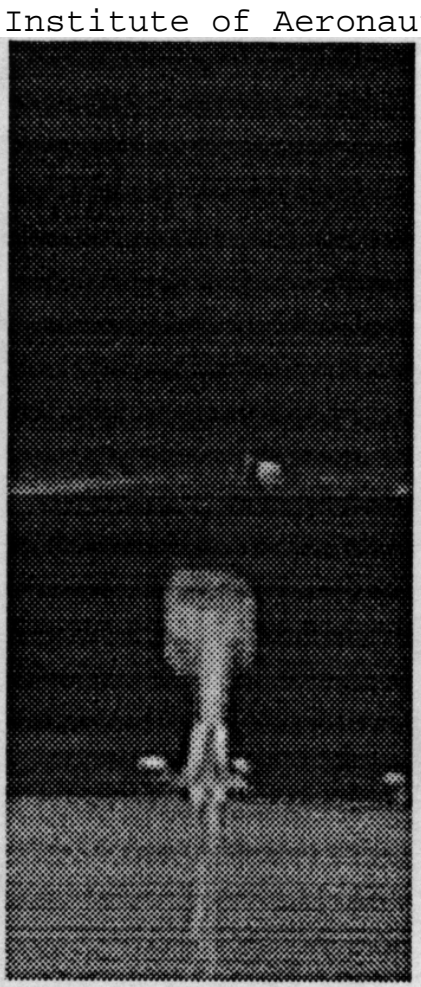

(b) $t=0.218 \mathrm{~s}$ and Astronautics, Inc.

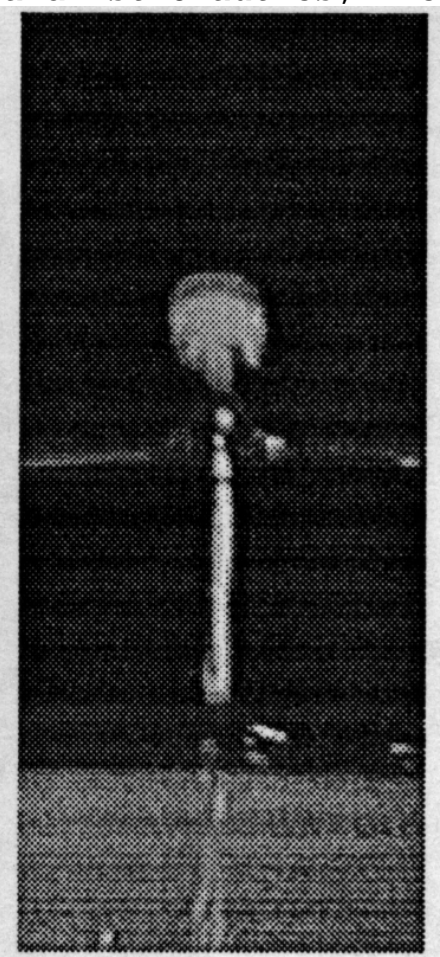

(c) $t=0.378 \mathrm{~s}$

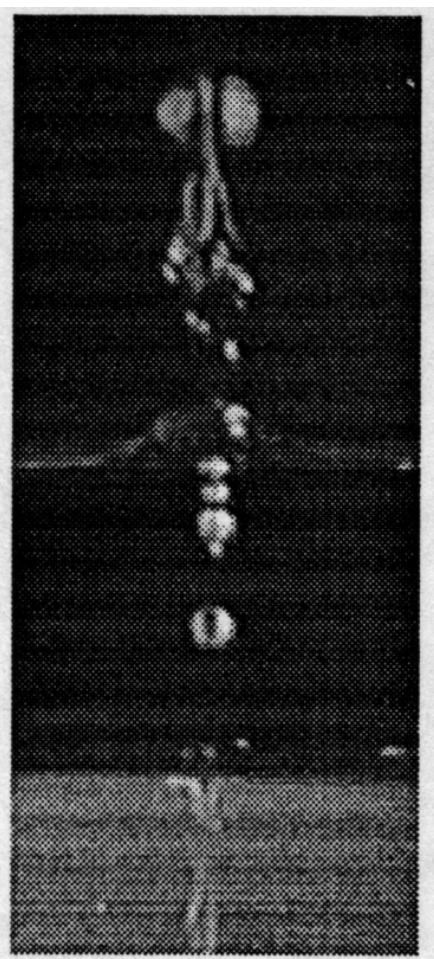

(d) $\mathrm{t}=0.587 \mathrm{~s}$

Fig. 2

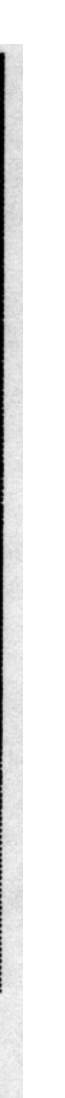




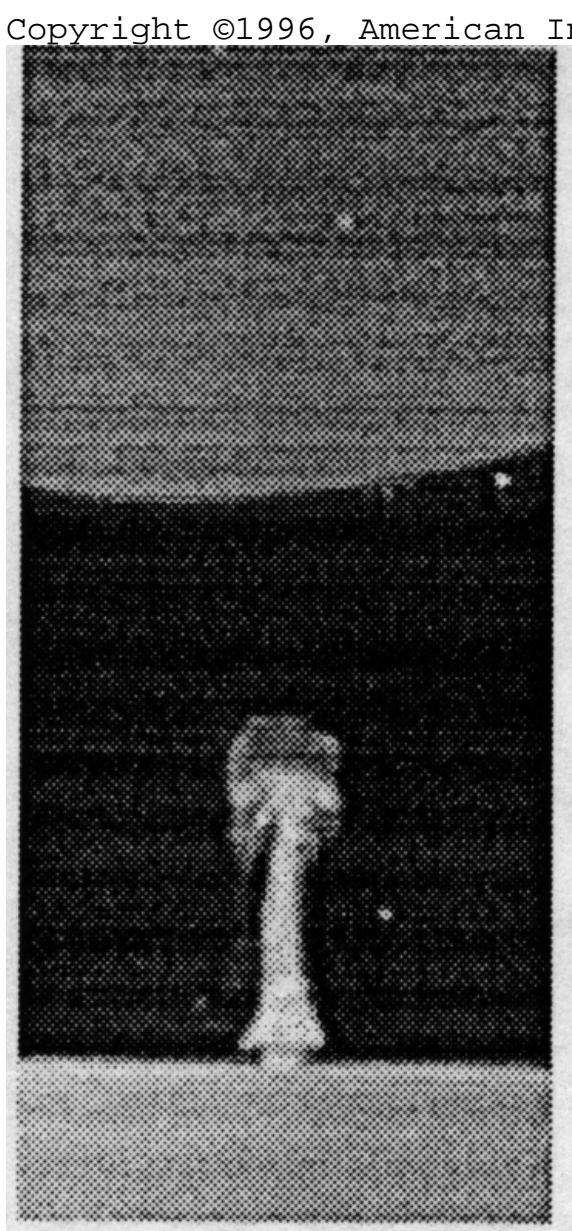

(a) $\mathrm{t}=0.158 \mathrm{~s}$

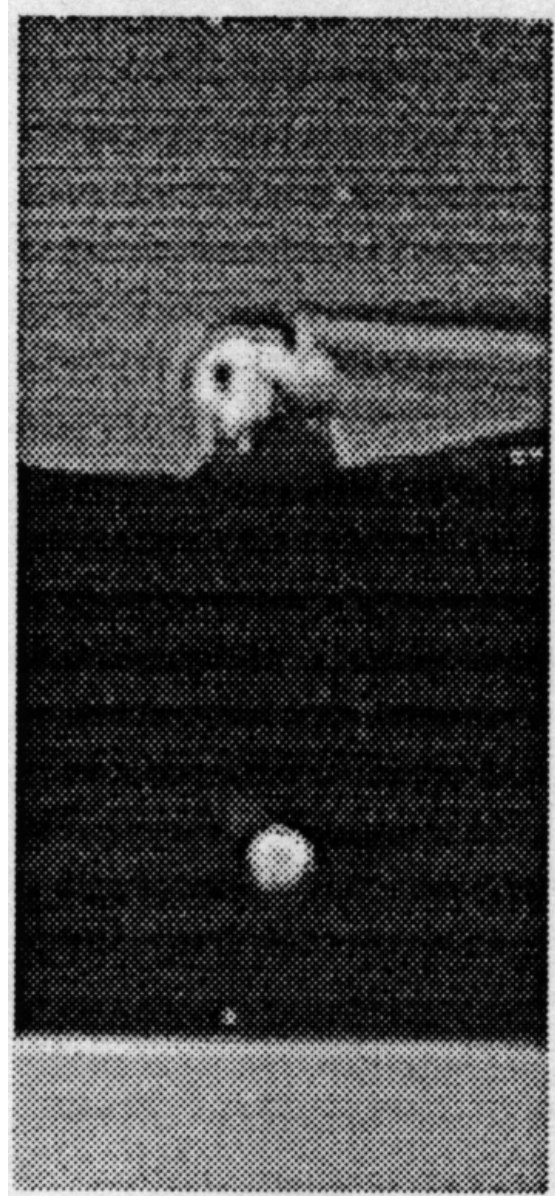

(d) $\mathrm{t}=0.458 \mathrm{~s}$

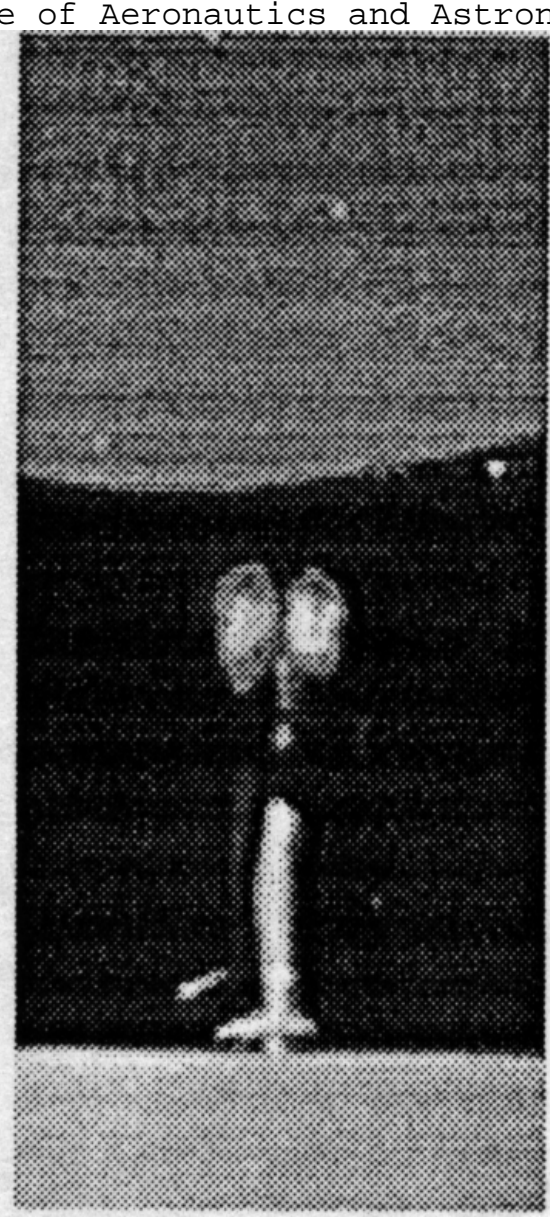

(b) $\mathrm{t}=0.218 \mathrm{~s}$

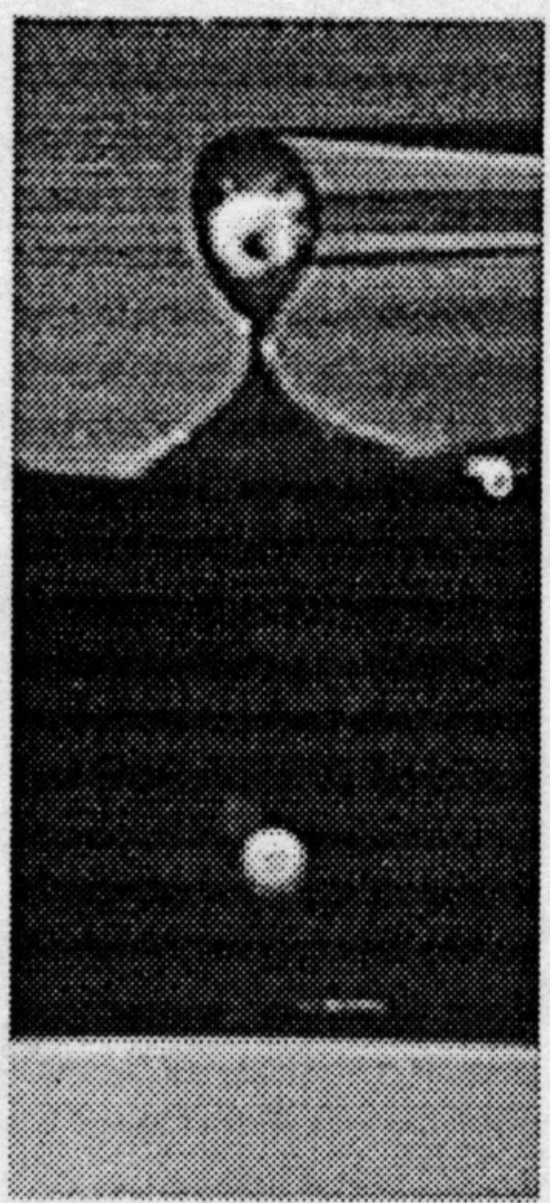

(e) $\mathrm{t}=0.988 \mathrm{~s}$

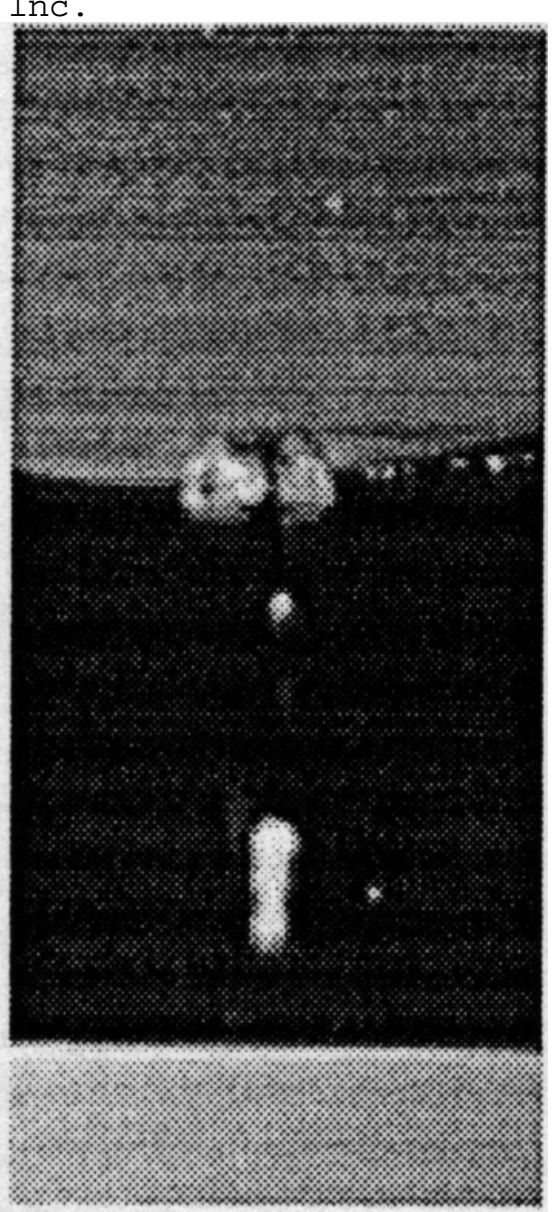

(c) $t=0.318 \mathrm{~s}$

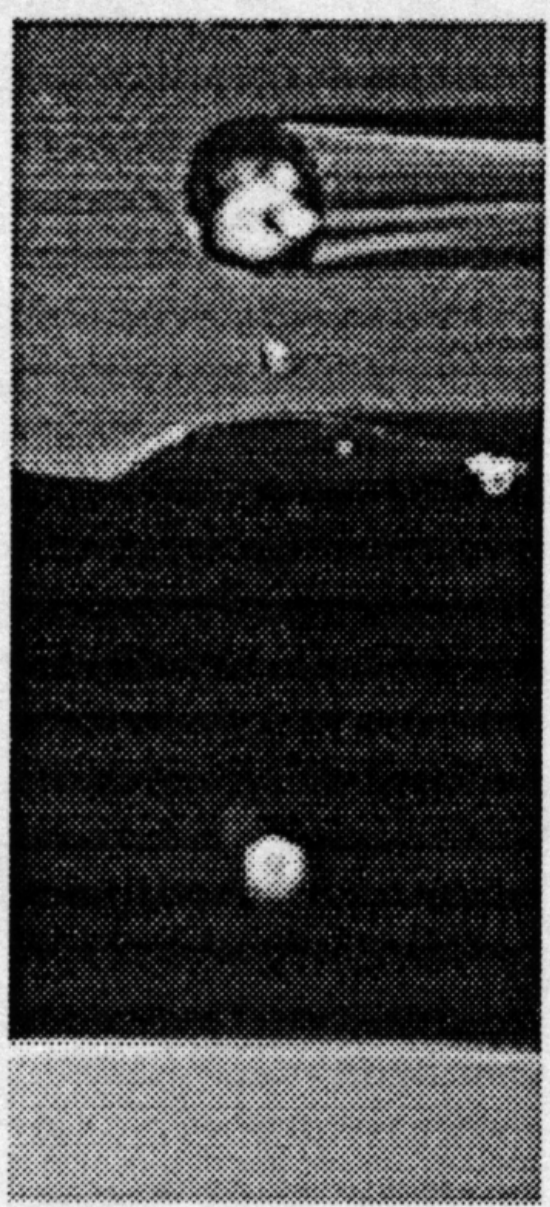

(f) $\mathrm{t}=1.118 \mathrm{~s}$ 